\title{
Quantitative Assessment of Blood Coagulation by Cold Atmospheric Plasma
}

\author{
Caitlin Heslin, ${ }^{a}$ Daniela Boehm, ${ }^{a}$ Vladimir Milosavljevic, ${ }^{a}$ Michelle Laycock, ${ }^{a}$ \\ PJ Cullen, a,b \& Paula Bourke ${ }^{a, *}$ \\ aPlasma Research Group, College of Science and Health, Dublin Insitute of Technology, Dublin \\ 1, Ireland; ' School of Chemical Engineering, University of New South Wales, Sydney, Australia \\ *Address all correspondence to: Paula Bourke, School Food Science and Environmental Health, Dublin Institute of \\ Technology, Dublin 1, Ireland; Email: paula.bourke@dit.ie.
}

\begin{abstract}
Cold atmospheric plasma (CAP) is produced by ionizing a chosen gas, thereby creating charged and reactive species. The reactive species generated are capable of inducing a range of biomedically relevant interactions including blood coagulation. However, the underlying biochemical processes of plasma-assisted blood coagulation are largely unknown, and data quantifying blood clot formation or the impact of system parameters on the intensity of the blood clot are scarce. In this study, blood coagulation was quantified by measuring hemoglobin absorbance. System parameters of the kINPen plasma jet were investigated and compared, including treatment time, distance from the plasma source and gas flow rate. These investigations were combined with optical emission spectroscopy to associate the species generated in the plasma effluent with the effect on coagulation efficiency. In this study, we have proposed a method to quantitatively assess plasma-induced blood coagulation to directly compare the clotting capabilities of other plasma systems and to explicate the mechanism of plasma-assisted blood coagulation.
\end{abstract}

KEY WORDS: Cold plasma, plasma jet, blood coagulation, plasma medicine, nonthermal plasma

\section{INTRODUCTION}

Cold atmospheric plasma (CAP) is an ionized gas that can be generated using different gases or gas mixtures, which consists of free electrons, radicals, and positively and negatively charged particles. ${ }^{1}$ The antimicrobial effects of CAP are well studied and have been proven to inactivate bacteria ${ }^{2,3}$ and fungi, ${ }^{4}$ and to have similarly cytotoxic effects on cancer cells. ${ }^{5} \mathrm{~A}$ range of CAPs have also been investigated for biomedical application in terms of wound healing and disinfection ${ }^{6,7}$ and have been proven to rapidly coagulate blood. $^{8-12}$

Coagulation is the formation of a blood clot and occurs when blood changes from a liquid to a solid. Coagulation is an important primary step of hemostasis and occurs immediately after damage to the endothelium of the blood vessel to prevent blood loss from the injured vessel and to seal the area from infection. ${ }^{13}$ Disruption of the endothelium activates platelets, causing them to adhere to the subendothelium, forming a plug. ${ }^{14}$ Simultaneously, secondary hemostasis is triggered; this is a complex cascade involving protein coagulation factors in the blood plasma culminating in the formation of fibrin strands forming a mesh to reinforce the platelet plug. ${ }^{15}$ The coagulation cascade requires 
calcium ions as a cofactor; coagulation factors use the ions to bind to the negatively charged phospholipid surface of platelets. ${ }^{16}$ Anticoagulants work by chelating calcium ions, causing conformational changes in the coagulation factors, which prevents activation of the coagulation cascade.

The release of chemokines from the clot and surrounding tissues stimulates leukocyte migration to the area to prevent infection and promote wound healing. ${ }^{17}$ Coagulation is highly conserved in vertebrates and involves both cellular (platelet) and protein (coagulation factor) components. ${ }^{16}$

Plasma-coagulation devices, such as the argon plasma coagulator (APC), are used in clinical medicine to ensure rapid coagulation. ${ }^{18,19}$ The APC is a high-frequency monopolar instrument used for thermal coagulation through cauterization and desiccation of the blood. ${ }^{20} \mathrm{CAP}$ would be ideal for bleeding control to stimulate the natural coagulation cascade with little damage to surrounding tissues. It has been previously demonstrated that anticoagulated blood samples can be clotted by CAP produced by a dielectric barrier discharge ${ }^{11}$ and plasma torches. ${ }^{21}$ There are multiple theories on the biochemical mechanisms of plasma-induced blood coagulation, such as activation of erythrocyte-platelet interactions and the coagulation cascade by reactive oxygen species (ROS) generated by CAP. ${ }^{11}$ Some studies have shown that platelet function and aggregation are influenced by oxygen species. ${ }^{22,23}$ There is also evidence to suggest that CAP has a direct effect on blood proteins, causing coagulation with no change to $\mathrm{Ca}^{2+}$ concentration or $\mathrm{pH}$ of the sample. ${ }^{11}$ Different studies have employed a range of plasma-generating systems and inducer gases. Thus, CAP has been shown to induce blood coagulation, but the underlying biochemical mechanisms and influence of process parameters remain poorly understood. Using a method based on specific absorbance of hemoglobin at 405 $\mathrm{nm}$, we analyzed plasma-induced blood coagulation in a quantitative manner and to relate clotting efficiency to different system parameters. In this study, we compared the intensity of blood clot formation in relation to different cold plasma jet process parameters by measuring hemoglobin absorbance as the biological response. We also utilized spectroscopic diagnostics of generated reactive species at the selected parameters and correlated them to the quantified blood clot to begin to elucidate the biochemical mechanisms of plasma-induced blood clot formation.

\section{MATERIALS AND METHODS}

\section{A. Plasma System}

The atmospheric plasma pressure jet (APPJ) kINPen Plasma MED (INP Greifswald/ neoplas tools GmbH Greifswald, Germany) was used for these investigations. This device is CE-certified as a medical device, meeting EU safety, health, and environmental regulations..$^{24,25}$ The apparatus consists of a hand-held unit, a DC power supply, and a gas supply unit. Plasma is generated in the pen and extends to the surrounding air outside the nozzle, and the discharge effluent is directed toward the sample. 
The handheld unit was mounted on a platform that controlled the vertical and horizontal movement of the jet to within $0.01 \mathrm{~mm}$. Argon gas was employed as a working gas as a noble, normally unreactive stable gas. ${ }^{24}$ For blood coagulation studies, process parameters studied using the kINPen jet included time, distance, and gas flow rate.

\section{B. Blood Collection and Preparation of Blood Components}

Anticoagulated blood samples were collected from the bovine coccygeal vein, under veterinarian supervision using sterile tubes containing anticoagulants EDTA or sodium citrate (BD Vacutainer $\left.{ }^{\circledR}\right)$ and was appropriately inverted according to supplier guidelines to ensure that the anticoagulant was correctly mixed throughout the sample. Samples were stored at $4{ }^{\circ} \mathrm{C}$ and processed within 12 hours of collection. The blood collection tubes were designed with maximum and minimum fill lines to draw the appropriate volume of blood to ensure a correct blood-to-additive ratio.

\section{Plasma-Induced Blood Clotting}

The plasma-induced blood clotting experiments were carried out in 96-well microtiter plates with $50-\mu 1$ blood samples undergoing plasma treatment. The negative control was a $50-\mu 1$ blood sample left in air for the same period of time. The positive control was $50 \mu$ blood treated with plasma for 10 minutes for a complete clot to be achieved with no liquid fraction.

\section{Quantification of Plasma-Induced Blood Clot}

Quantification of blood coagulation was achieved by adapting the Harboe spectrophotometric assay for the measurement of hemoglobin, using absorbance measurement at $405 \mathrm{~nm}^{26}$ in a microtiter plate reader. The treated blood sample was washed with PBS (Sigma-Aldrich, Dublin, Ireland), and the unclotted blood portion was separated from the formed blood clot by pipetting it into an Eppendorf tube and acidifying the solution to achieve a 3\% acetic acid solution (Sigma-Aldrich, Dublin, Ireland). The remaining blood clot was separately dissolved using 3\% acetic acid in PBS, and the well was washed three times with $3 \%$ acetic acid to remove any residual hemoglobin, then it was transferred to a separate Eppendorf tube. Both the unclotted fraction and dissolved blood clot were appropriately diluted, and absorbance readings were recorded using a Synergy HT microplate reader (Biotek, Potton, UK). These results were compared to an untreated wholeblood sample as well as a control blood clot, respectively, to determine a blood clotting index (BCI) as described in Eq.1, similar to that described by Wang. ${ }^{27}$

$$
\text { BCI }(\%)=\quad \text { Absorbance of acidified whole blood/control clotted blood } \mathrm{X} 100
$$




\section{E. Optical Emission Spectroscopy}

Optical emission spectroscopy (OES) was recorded using an optical fiber spectrometer (Edmund Optics, York, UK) in the ultraviolet (UV), visible (VIS), and near-infrared (NIR) regions, including the emission of $\mathrm{OH}$ radicals at $309 \mathrm{~nm}$ and the excited argon species at $750 \mathrm{~nm}$ and $811 \mathrm{~nm}$. The plasma images were taken along the plasma plume while treating the sample, with spatial resolution of $0.01 \mathrm{~mm}$. The spectrometer was calibrated against a halogen lamp.

\section{RESULTS}

CAP has been shown to induce blood coagulation, but the underlying biochemical mechanisms and influence of plasma parameters remain poorly understood. Using a method based on the specific absorbance of hemoglobin at $405 \mathrm{~nm}$, we analyzed plasma-induced blood coagulation in a quantitative manner and attempted to relate clotting efficiency to different system parameters.

The system parameters applied included treatment time, distance from the plasma source, and gas flow rate. These factors were found to effect blood clot formation and were correlated to OES data to elucidate the influence of plasma on the coagulation cascade.

\section{A. Treatment Time}

The optical emission spectra of the argon plasma discharged in air (Fig. 1a) shows that the overall emission intensity, an indication of the energy in the plasma system, stabilized over time. The individual spectra of the wavelengths of interest, namely excited argon at $750 \mathrm{~nm}$ and $811 \mathrm{~nm}$ and the hydroxyl radical $(\mathrm{OH})$ emission at 309 $\mathrm{nm}$, also showed stabilization over the treatment time (Fig. 1b). The ratio of the spectral lines recorded for the excited argon species is proportional to the electron energy distribution factor (EEDF) and is a mean value of electron energy. ${ }^{28}$ The intensity of the plasma was demonstrated to stabilize over time using OES, and treatment times in excess of 2 min were used for more reproducible results. The intensity of $\mathrm{OH}$ emission at $309 \mathrm{~nm}$ was higher than the argon emission. Optical emission spectra were recorded $2 \mathrm{~mm}$ above the sample, with the jet at a distance of $18 \mathrm{~mm}$ from the sample and a gas flow rate of $2.5 \mathrm{~cm}^{3} / \mathrm{s}$. The percentage of CAP-clotted blood increased with treatment time (Fig. 2).

When taking the OES spectra over treatment time and comparing them to the effect of distance from the plasma jet on the plasma intensity (Fig. 3), we observed interactive effects. Although there was an increase in plasma-induced blood clotting in conjunction with treatment time and the plasma intensity was stable, the intensity of the plasma reaching the sample decreased with increasing distance. 


\section{Distance from the Sample}

As the plasma jet distance from the blood sample was increased at a fixed treatment time of 4 minutes and gas flow rate of $2.5 \mathrm{~cm}^{3} / \mathrm{s}$, there was a marked reduction in plasma-induced clotting. Blood clotting decreased linearly with an increase in distance from the plasma source, as shown in Fig. 4(a), and the captured emission spectra of the reactive species reaching the sample also indicated overall decrease with distance (Fig. 4b). These data suggest a relationship between blood coagulation and short-lived reactive species in the plume, which are dispersed, resulting in a decrease in the intensity of the plume reaching the sample (Fig. 4c).
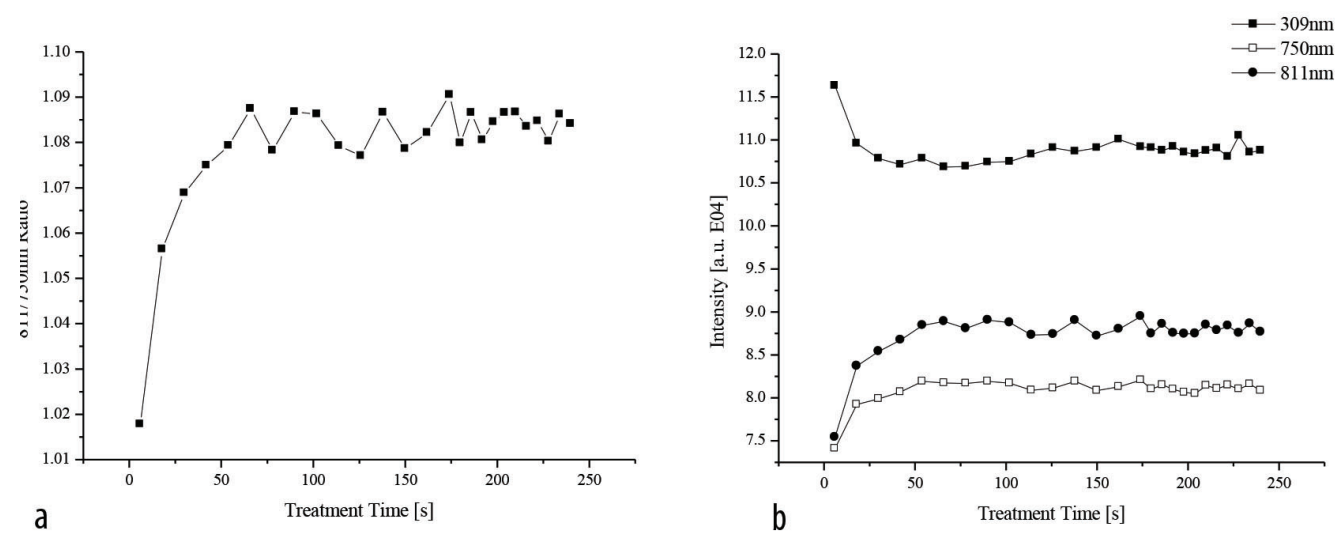

FIG. 1 (a): Overall intensity recorded with OES diagnostics of the argon plasma jet over time; (b): Intensity recorded at $309 \mathrm{~nm}, 750 \mathrm{~nm}$, and $811 \mathrm{~nm}$, respectively

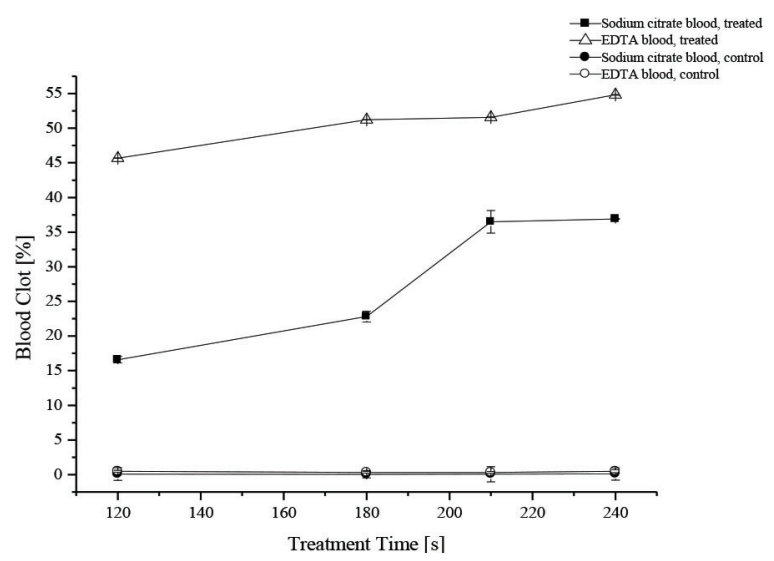

FIG. 2: The effect of time on clot formation in anticoagulated blood treated with the plasma jet 


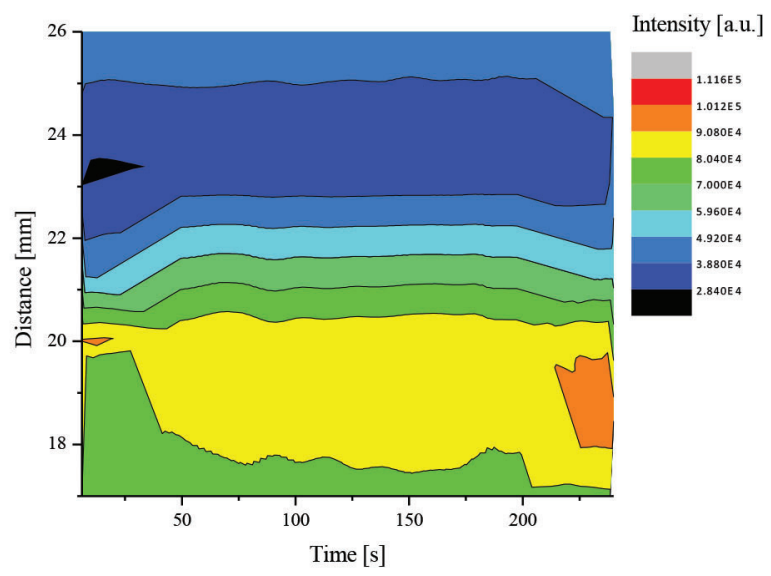

FIG. 3: The effect of treatment time and distance on overall plasma intensity

\section{Gas Flow Rate}

The intensity of the plasma plume stabilized with increasing gas flow rate after an initial increase (Fig. 5a). No reactive species were generated with a gas flow rate $<1.5 \mathrm{~cm}^{3} / \mathrm{s}$. This stability in emission led to treatment at a gas flow rate of $2.5 \mathrm{~cm}^{3} / \mathrm{s}$. With a gas flow rate $>3.5 \mathrm{~cm}^{3} / \mathrm{s}$, there was visible dispersion of the sample due to the gas pressure. Emission spectra of the hydroxyl radical at $309 \mathrm{~nm}$ should have offered a similar pattern; however, we observed a decrease in the hydroxyl radical reaching the sample (Fig. 5b), due to a possible quenching of species by the sample and resulting increase of the blood clot (Fig. 5c). Therefore, the ideal gas flow rate and treatment time are shown in Fig. 6. The highest plasma intensity coincides with a gas flow rate of $1.9-2.4 \mathrm{~cm}^{3} / \mathrm{s}$ and a treatment time of 4 minutes.

\section{DISCUSSION}

Cold plasmas have been investigated for decades, but research into their use as selective therapeutic instruments is relatively recent. Employment of surgical plasma technology is already in operation with the APC based on ablation and dessication. An alternative therapy, which could rapidly coagulate during surgery or upon injury and offer little to no collateral damage, would be of great importance to the medical field. Different CAP systems, including the air plasma torch ${ }^{12,21}$ and floating-electrode dielectric barrier discharge, ${ }^{9,10,29}$ have proven to rapidly coagulate blood in vitro and in vivo with no effect to the surrounding tissues. ${ }^{9,29-37}$ A method of comparison between systems is needed to assess different treatment parameters such as time and distance from the plasma source. In the present study, we demonstrated a method for comparing the clotting capabilities of different CAP systems. In previous studies, the blood clotting ability of CAP has only been assessed qualitatively using visual evaluation. ${ }^{8,10,11}$ 

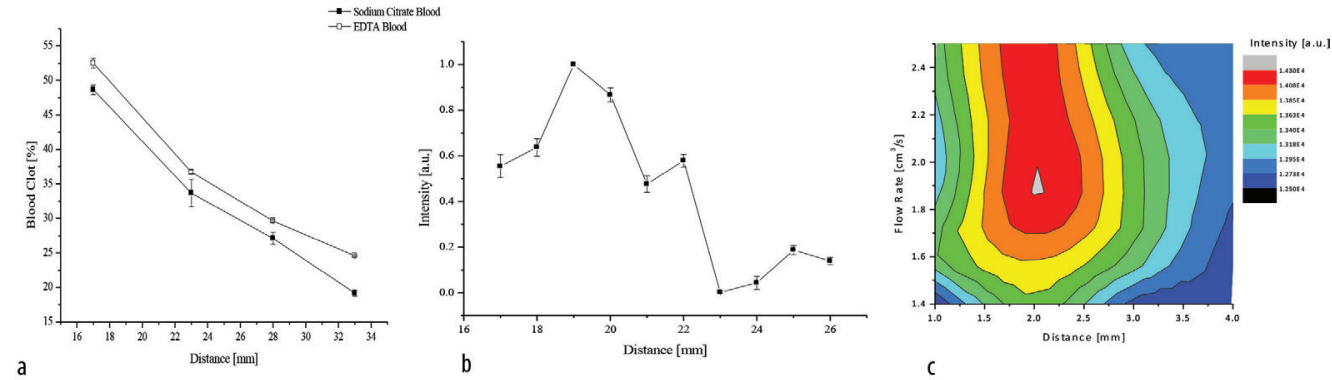

FIG. 4: The effect of distance from plasma source (a) on blood clot formation; (b) the effect of distance on the intensity of plasma plume; (c) the combined effect of gas flow rate and distance on plasma intensity
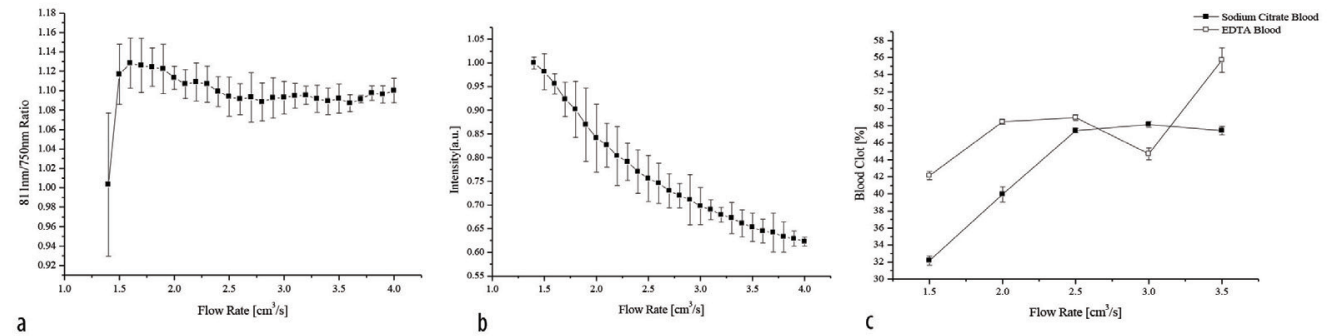

FIG. 5: The effect of gas flow rate on (a) intensity of the argon plasma; (b) emission spectra of the hydroxyl radical at $309 \mathrm{~nm}$; (c) blood clot formation

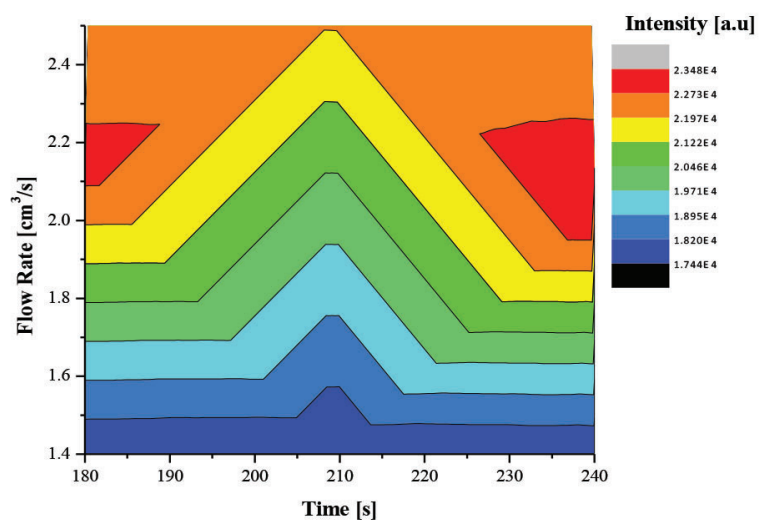

FIG. 6: The effect of treatment time and gas flow rate on the intensity of the plasma jet 
Quantitative assessment of blood coagulation using an argon plasma jet was conducted to compare clotting efficiency to the system parameters of distance from plasma source, gas flow rate, and treatment time. The plasma was characterized using optical emission spectroscopy. Our results suggest an important role for short-lived oxygen plasma species, such as the hydroxyl radical, in initiating blood coagulation. Reactive oxygen species, such as hydrogen peroxide and hydroxyl radical, ${ }^{38,39}$ have been implicated in influencing the coagulation cascade via the activation of platelets. ${ }^{40,41}$ It has been suggested that ROS cause clotting by inactivating anticoagulation agents that control clotting, such as endothelial-derived relaxing factor (EDRF), which counteracts platelet aggregation in vivo. ${ }^{40}$ However, treatment time and gas flow rate also effect blood coagulation but they ultimately plateau, pointing to optimal parameter selection for coagulation. The diminution in blood-clot formation with increasing distance between the plasma plume and the blood sample was attributed to the reduction of reactive species transmitted to the sample. Integrating these results with the quantitative assessment of blood clot formation, we were able to optimize the distance from the sample in conjunction with treatment time and the gas flow rate applied to the device.

The blood samples used in this experiment included two different types of anticoagulants that sequester calcium ions in the blood, thus preventing their involvement in the coagulation cascade and use by coagulation factors in binding to phospholipid surfaces. ${ }^{16}$ Sodium citrate chelates the calcium ions in blood, but this process can be reversed through the addition of calcium ions to the sample. EDTA irreversibly binds the calcium ions in blood and prevents their influence in the coagulation cascade, thereby preventing the blood from forming clots. Our results revealed a tendency of the EDTA blood to exhibit better clot formation under CAP treatment. The clotting of anticoagulated blood, and of EDTA-treated blood in particular, with CAP suggests that plasma-assisted coagulation is not dependent on $\mathrm{Ca}^{2+}$ ions. These findings are similar to those of Kalghatgi et al. ${ }^{11}$

\section{CONCLUSIONS}

The ability of CAP to rapidly coagulate anticoagulated blood in a matter of minutes indicates the importance of this tool in a medical scenario to prevent hemorrhaging and to seal a wound from infection.

The biochemical mechanisms whereby the coagulation cascade is activated by CAP remain to be elucidated, but the methods employed here can be used not only to compare coagulation capabilities associated with different process parameters but also to directly quantitatively compare coagulation capabilities across different systems to explain and optimize the cascade activation mechanism. These results also point to the enhanced role of some short-lived reactive species, suggesting an opportunity to maximize coagulative effects through varying process parameters such as inducer gas. 


\section{ACKNOWLEDGMENTS}

This research was funded through Science Foundation Ireland under approval code 11/ TIDA/B1928. We especially thank veterinarian Dr. David Fagan of Mullingar Pet Hospital, for his help with collecting bovine blood.

\section{REFERENCES}

1. Kong MG, Kroesen G, Morfill G, Nosenko T, Shimizu T, Van Dijk J, Zimmermann JL. Plasma medicine: an introductory review. New J Phy. 2009;11:115012.

2. Lu H, Patil S, Keener KM, Cullen PJ, Bourke P. Bacterial inactivation by high-voltage atmospheric cold plasma: influence of process parameters and effects on cell leakage and DNA. J Appl Microbiol. 2013;116:784-94.

3. Ziuzina D, Patil S, Cullen PJ, Keener KM, Bourke P. Atmospheric cold plasma inactivation of Escherichia coli in liquid media inside a sealed package. J Appl Microbiol. 2013;114:778-87.

4. Kuo SP, Chen C-Y, Lin C-S, Chiang S-H. Applications of air plasma for wound bleeding control and healing. IEEE Trans Plasma Sci. 2012;40:1117-23.

5. Zhao S, Xiong Z, Mao X, Meng D, Lei Q, Li Y, Deng P, Chen M, Tu M, Lu X, Yang G, He G. atmospheric pressure room temperature plasma jets facilitate oxidative and nitrative stress and lead to endoplasmic reticulum stress dependent apoptosis in HepG2 cells. PLoS One. 2013;8:e73665.

6. Isbary G, Heinlin J, Shimizu T, Zimmermann JL, Morfill G, Schmidt HU, Monetti R, Steffes B, Bunk W, Li Y, Klaempfl T, Karrer S, Landthaler M, Stolz W. Successful and safe use of 2 min cold atmospheric argon plasma in chronic wounds: results of a randomized controlled trial. Br J Dermatol. 2012;167:404-10.

7. Arndt S, Unger P, Wacker E, Shimizu T, Heinlin J, Li YF, Thomas HM, Morfill GE, Zimmermann JL, Bosserhoff AK, Karrer S. Cold atmospheric plasma (CAP) changes gene expression of key molecules of the wound healing machinery and improves wound healing in vitro and in vivo. PLoS One. 2013;8:e79325.

8. Chen C-Y, Fan H-W, Kuo SP, Chang J, Pedersen T, Mills TJ, Huang C-C. Blood clotting by low temperature air plasma. IEEE Trans Plasma Sci. 2009;37(6):993-99.

9. Dobrynin D, Wasko K, Friedman G, Fridman A, Fridman G. Fast blood coagulation of capillary vessels by cold plasma: a rat ear bleeding model. Plasma Medicine. 2011;1(3-4):241-7.

10. Fridman G, Peddinghaus M, Balasubramanian M, Ayan H, Fridman A, Gutsol A, Brooks A. Blood coagulation and living tissue sterilization by floating-electrode dielectric barrier discharge in air. Plasma Chem Plasma Pro. 2006;26:425-42.

11. Kalghatgi SU, Fridman G, Cooper M, Nagaraj G, Peddinghaus M, Balasubramanian M, Vasilets VN, Gutsol AF, Fridman A, Friedman G. Mechanism of blood coagulation by nonthermal atmospheric pressure dielectric barrier discharge plasma. IEEE Trans Plasma Sci. 2007;35(5, part 2):1559-66.

12. Kuo SP, Tarasenko O, Chang J, Popovic S, Chen CY, Fan HW, Scott A, Lahiani M, Alusta P, Drake JD, Nikolic M. Contribution of a portable air plasma torch to rapid blood coagulation as a method of preventing bleeding. New J Phys. 2009;11:1150156

13. Austin SK. Haemostasis. Medicine. 2013;41:208-11.

14. Norris LA. Blood coagulation. Best Pract Res Clin Obstet Gynaecol. 2003;17(3):369-83.

15. Eyre L, Gamlin F. Haemostasis, blood platelets and coagulation. Anaesth Intensive Care. 2010;11:2446.

16. Spronk HM, Govers-Riemslag JW, ten Cate H. The blood coagulation system as a molecular machine. Bioessays. 2003;25:1220-8.

17. Guo S, DiPietro L. Factors affecting wound healing. J Dent Res. 2010;89:219-29.

18. Vargo JJ. Clinical applications of the argon plasma coagulator. Gastrointest Endosc. 2004;59:81-8. 
19. Malick KJ. Clinical applications of argon plasma coagulation in endoscopy. Gastroenterol Nurs. 2006;29:386-91.

20. Farin G, Grund KE. Technology of argon plasma coagulation with particular regard to endoscopic applications. Endosc Surg Allied Technol. 1994;2:71-7.

21. Kuo SP, Chen C-Y, Lin C-S, Chiang S-H. Wound bleeding control by low temperature air plasma. IEEE Trans Plasma Sci. 2010;38(8, part 2):1908-14.

22. Stief TW, Kurz J, Doss MO, Fareed J. Singlet oxygen inactivates fibrinogen, factor V, factor VIII, factor X, and platelet aggregation of human blood. Thromb Res. 2000;97:473-80.

23. Del Principe D, Menichelli A, De Matteis W, Di Corpo ML, Di Giulio S, Finazzi-Agro A. Hydrogen peroxide has a role in the aggregation of human platelets. FEBS Lett. 1985;185:142-6.

24. Weltmann K-D, Woedtke TV. Basic requirements for plasma sources in medicine. Eur Phys J Appl Phys 2011; 55:13807.

25. von Woedtke T, Metelmann HR, Weltmann KD. Clinical plasma medicine: state and perspectives of in vivo application of cold atmospheric plasma. Contrib Plasma Phys. 2014;54:104-17.

26. Harboe M. A method for determination of hemoglobin in plasma by near-ultraviolet spectrophotometry. Scand J Clin Lab Invest. 1959;11:66-70.

27. Wang QZ, Chen XG, Li ZX, Wang S, Liu CS, Meng XH, Liu CG, Lv YH, Yule J. Preparation and blood coagulation evaluation of chitosan microspheres. J Mater Sci Mater Med. 2008;19:1371-7.

28. Zhu XM, Pu YK, Balcon N, Boswell R. Measurement of the electron density in atmospheric-pressure low-temperature argon discharges by line-ratio method of optical emission spectroscopy. J Phys D: Appl Phys. 2009;42(142003).

29. Kalghatgi S, Kelly CM, Cerchar E, Torabi B, Alekseev O, Fridman A, Friedman G, Azizkhan-Clifford J. Effects of non-thermal plasma on mammalian cells. PLoS One. 2011;6:e16270.

30. Blackert S, Haertel B, Wende K, von Woedtke T, Lindequist U. Influence of non-thermal atmospheric pressure plasma on cellular structures and processes in human keratinocytes (HaCaT). J Dermat Sci. 2013;70:173-81.

31. Walter MNM, Wright KT, Fuller HR, MacNeil S, Johnson WEB. Mesenchymal stem cell-conditioned medium accelerates skin wound healing: an in vitro study of fibroblast and keratinocyte scratch assays. Exper Cell Res. 2010;316:1271-81.

32. Wende K, Landsberg K, Lindequist U, Weltmann KD, von Woedtke T. Distinctive activity of a nonthermal atmospheric-pressure plasma jet on eukaryotic and prokaryotic cells in a cocultivation approach of keratinocytes and microorganisms. IEEE Trans Plasma Sci. 2010;38:2479-85.

33. García-Alcantara E, López-Callejas R, Morales-Ramírez PR, Peña-Eguiluz R, Fajardo-Muñoz R, Mercado-Cabrera A, Barocio SR, Valencia-Alvarado R, Rodríguez-Méndez BG, Muñoz-Castro AE, Piedad-Beneitez Adl, Rojas-Olmedo IA. Accelerated mice skin acute wound healing in vivo by combined treatment of argon and helium plasma needle. Arch Med Res. 2013;44:169-77.

34. Isbary G, Köritzer J, Mitra A, Li YF, Shimizu T, Schroeder J, Schlegel J, Morfill GE, Stolz W, Zimmermann JL. Ex vivo human skin experiments for the evaluation of safety of new cold atmospheric plasma devices. Clin Plasma Med. 2013;1:36-44.

35. Lopes BB, Kraft MBdPL, Rehder J, Batista FRX, Puzzi MB. The interactions between non-thermal atmospheric pressure plasma and ex-vivo dermal fibroblasts. Procedia Engineering. 2013;59:92-100.

36. Ermolaeva SA, Varfolomeev AF, Chernukha MY, Yurov DS, Vasiliev MM, Kaminskaya AA, Moisenovich MM, Romanova JM, Murashev AN, Selezneva, II, Shimizu T, Sysolyatina EV, Shaginyan IA, Petrov OF, Mayevsky EI, Fortov VE, Morfill GE, Naroditsky BS, Gintsburg AL. Bactericidal effects of non-thermal argon plasma in vitro, in biofilms and in the animal model of infected wounds. J Med Microbiol. 2011;60(Pt 1):75-83.

37. Wu AS, Kalghatgi S, Dobrynin D, Sensenig R, Cerchar E, Podolsky E, Dulaimi E, Paff M, Wasko K, Arjunan KP, Garcia K, Fridman G, Balasubramanian M, Ownbey R, Barbee KA, Fridman A, Friedman G, Joshi SG, Brooks AD. Porcine intact and wounded skin responses to atmospheric nonthermal plasma. J Surg Res. 2013;179:e1-e12. 
38. Iuliano L, Pedersen JZ, Pratico D, Rotilio G, Violi F. Role of hydroxyl radicals in the activation of human platelets. Eur J Biochem. 1994;221:695-704.

39. Pretorius E, Bester J, Vermeulen N, Lipinski B. Oxidation inhibits iron-induced blood coagulation. Curr Drug Targets. 2013;14:13-9.

40. Ambrosio G, Tritto I, Golino P. Reactive oxygen metabolites and arterial thrombosis. Cardiovasc Res. 1997;34:445-52.

41. Kvietys PR, Granger DN. Role of reactive oxygen and nitrogen species in the vascular responses to inflammation. Free Radic Biol Med. 2012;52:556-92. 
